\title{
Originals
}

\section{Glyburide crosses the placenta in vivo in pregnant rats}

\author{
E.Sivan ${ }^{1}$, B. Feldman ${ }^{1}$, M.Dolitzki ${ }^{1}$, N.Nevo ${ }^{2}$, N. Dekel ${ }^{2}$, A.Karasik ${ }^{1}$ \\ ${ }^{1}$ Department of Obstetrics and Gynecology and Institute of Endocrinology, Sheba Medical Center, Tel Hashomer, Israel \\ ${ }^{2}$ Department of Hormone Research, Weizman Institute of Science, Rehovot, Israel
}

Summary Non-insulin-dependent diabetes mellitus (NIDDM) is normally treated by oral hypoglycaemic agents, but their use is excluded during pregnancy because of their potential teratogenic and hypoglycaemic effects on the fetus. This caveat was recently questioned as glyburide was shown to cross an isolated cotyledon in vitro in insignificant amounts. In the present study, placental transport of glyburide in vivo was examined as an indispensable step towards clinical trials. Tritiated glyburide, $\mathrm{C}^{14}$ albumin or $C^{14}$-labelled diazepam were injected into 13,9 and 11 pregnant rats, respectively and the radioactivity was measured thereafter in maternal blood and in whole fetal extracts. The ratios between radioactivity in fetal tissue to that in maternal blood for glyburide $(0.535 \pm 0.068)$ were similar to those of diazepam $(0.641 \pm 0.057)$ which readily crosses the pla- centa. However, they differed significantly from those for albumin $(0.048 \pm 0.0004)$ which does not cross. Moreover, glyburide in fetal tissue consistently reflected its concentration in maternal blood when measured at consecutive intervals after intravenous injection in the mother. In contrast, albumin in fetal tissue was low at all time points regardless of its levels in maternal blood when measured at different times after injection. These data suggest that glyburide crosses the placenta of pregnant rats and should therefore be considered with caution as a hypoglycaemic agent in the treatment of gestational diabetes. [Diabetologia (1995) 38: 753-756]

Key words Glyburide, oral hypoglycaemic agents, pregnancy.
The majority of women suffering from gestational diabetes show carbohydrate intolerance due to peripheral resistance to insulin, which resembles noninsulin-dependent diabetes mellitus (NIDDM). Although NIDDM is usually treated by oral hypoglycaemic agents (OHA) [1], insulin is the only accepted mode of therapy when dietary means fail to maintain euglycaemia during pregnancy [2]. OHAs are considered unsafe for use during pregnancy because of potential teratogenicity and pro-

Received: 9 June 1994 and in revised form: 6 December 1994

Corresponding author: Dr. A. Karasik, Institute of Endocrinology, The Chaim Sheba Medical Center, Tel Hashomer 52621 Israel

Abbreviations: NIDDM, Non-insulin-dependent diabetes mellitus; OHA, oral hypoglycaemic agents. longed neonatal hypoglycaemia [3-7]. However, published studies on OHAs in diabetic pregnant women have so far been scanty and somewhat confusing [8-12].

Were it possible to show that an OHA did not pass through the placental barrier, such an agent could be safely administered during pregnancy, with the obvious advantages of this mode of therapy. Elliot et al. [13] have used an in vitro, isolated, single human cotyledon placental model, in order to study the placental transport of glyburide. They conclude that only insignificant amounts of glyburide cross the human placenta.

The present study was designed to assess the in vivo transport of glyburide across the placenta of pregnant rats, as an indispensable step towards clinical trials. To this end pregnant rats were injected with labelled glyburide, diazepam or albumin. Diaz- 
epam was chosen as a control as it shares similar protein binding, partition coefficients and molecular weight with glyburide and readily crosses the placental barrier. Albumin, with a molecular weight of 69,000 , does not cross the placenta and thus was the other choice as control. Distribution of all three drugs between mother and fetus was then evaluated and compared.

\section{Materials and methods}

Animals. Four- to six-month-old pregnant Wistar rats weighing 270-300 g were used throughout the experiments. To establish pregnancy and determine the exact gestational age, daily vaginal smears were examined. The day of conception was considered day 1 of gestation. Pharmacokinetic studies were all performed on days $14-16$ of gestation.

Radiolabelled materials. Tritiated glyburide (specific activity $48.5 \mu \mathrm{Ci} / \mathrm{mmol}$ ) and $\mathrm{C}^{14}$-labelled methylated albumin (specific activity $0.0223 \mathrm{mCi} / \mathrm{mg}$ ) were obtained from New England $\mathrm{Nu}-$ clear (Dreiech, Germany). $\mathrm{C}^{14}$-labelled diazepam (specific activity $53 \mu \mathrm{Ci} / \mathrm{mmol}$ ) was provided by Amersham (Amersham Bucks., UK). Radiochemical purity of glyburide and diazepam was found to be $99 \%$ (using high-performance liquid chromatography) and $98.4 \%$ for albumin (by gel electrophoresis), respectively.

\section{Experimental design}

Measurement of radioactivity at a specific time post injection. Ten pregnant rats were given a single intraperitoneal injection of tritiated glyburide (total dose $20 \mu \mathrm{Ci}=200 \mathrm{ng}$ ) dissolved in dimethyl sulphoxide (DMSO) $25 \%$, Tween-80 $5 \%$ and isotonic saline $70 \%$. Sixty min later animals were anaesthetized and bled by heart puncture into heparinized glass tubes. The maternal abdomen was immediately opened and the uterine horns with fetuses and placentas removed. After washing with isotonic saline, they were isolated and weighed. The saline was measured for radioactivity in order to detect contamination with maternal blood. Whole fetuses and placentas were then homogenized with a polytron blender. Tissue homogenates and maternal sera were placed in scintillation vials and total radioactivity was determined for each sample using a beta-counter (Beckman LS 5000 TD, Palo Alto, Ca, USA).

To ensure that all radioactivity delivered to fetuses was blood-borne when injected intraperitonealy, an additional three animals were given radiolabelled glyburide intravenously, and the results were compared.

Nine rats injected intraperitoneally with $\mathrm{C}^{14}$-labelled albu$\min$ (total dose $0.1 \mathrm{mCi}$ ) using the identical protocol and eleven rats injected with intraoperitoneal $\mathrm{C}^{14}$-labelled diazepam (total dose $2 \mu \mathrm{Ci}$ ) were used as controls. Radioactivity was measured and evaluated in the same manner.

The results are expressed as $\mathrm{dpm} / \mathrm{g}$ wet tissue weight divided by dpm in $1 \mathrm{ml}$ of maternal blood for all drugs.

\footnotetext{
Measurement of radioactivity at different time intervals post injection. Two groups of eight pregnant rats were given a single injection of glyburide or albumin. At each time point between 20 and 225 min, two rats were anaesthetized and examined as described.
}

Table 1. Experiments performed at a specific time post injection

\begin{tabular}{llrl}
\hline Material & Administration & No. animals & $\begin{array}{l}\text { Fetal/ } \\
\text { maternal ratio }\end{array}$ \\
\hline Glyburide & Intraperitoneal & 10 & $0.541 \pm 0.077$ \\
Glyburide & Intravenous & 3 & $0.515 \pm 0.143$ \\
Albumin & Intraperitoneal & 9 & $0.048 \pm 0.0004$ \\
Diazepam & Intraperitoneal & 11 & $0.641 \pm 0.057$ \\
\hline
\end{tabular}

Simultaneous injection of radiolabelled glyburide and albumin. To exclude the possibility that the results of placental transport of glyburide reflect the influence of solvents or differences in placental reaction of the different animals used, tritiated glyburide and $\mathrm{C}^{14}$-labelled albumin were simultaneously injected intraperitoneally and intravenously, respectively. The three injected animals who were on their 16th gestational day were examined as described in the first set of experiments.

The ratio between radioactivity in fetal tissue and in maternal blood was determined and the results were compared, for both substances.

\section{Statistical analysis}

Data were analysed with the Student's $t$-test.

\section{Results}

\section{Measurement of radioactivity at a specific time post injection}

The ratios between radioactivity in fetal tissue to that in maternal blood are given in Table 1 . The results for glyburide were pooled as they were very close for both routes of administration $(0.541 \pm$ 0.77 vs $0.515 \pm 0.143$ for intraperitoneal and intravenous, respectively). The glyburide distribution ratio $(0.535 \pm 0.068)$ was similar to that for diazepam $(0.641 \pm 0.057)$ which readily crosses the placenta, but differed significantly from that of albu$\min (0.048 \pm 0.0004, p<0.001))$ which does not cross.

\section{Measurement of radioactivity at different time intervals post injection}

Results of the time-related experiments indicate that the concentrations of radioactive glyburide in fetal tissue consistently reflect its concentrations in maternal blood (Fig.1). On the other hand, radioactivity of labelled albumin in fetal tissue was consistently low regardless of its high levels found in maternal blood (Fig. 2). These results indicate that glyburide crosses the placenta in a manner reflecting its concentration in maternal blood. 


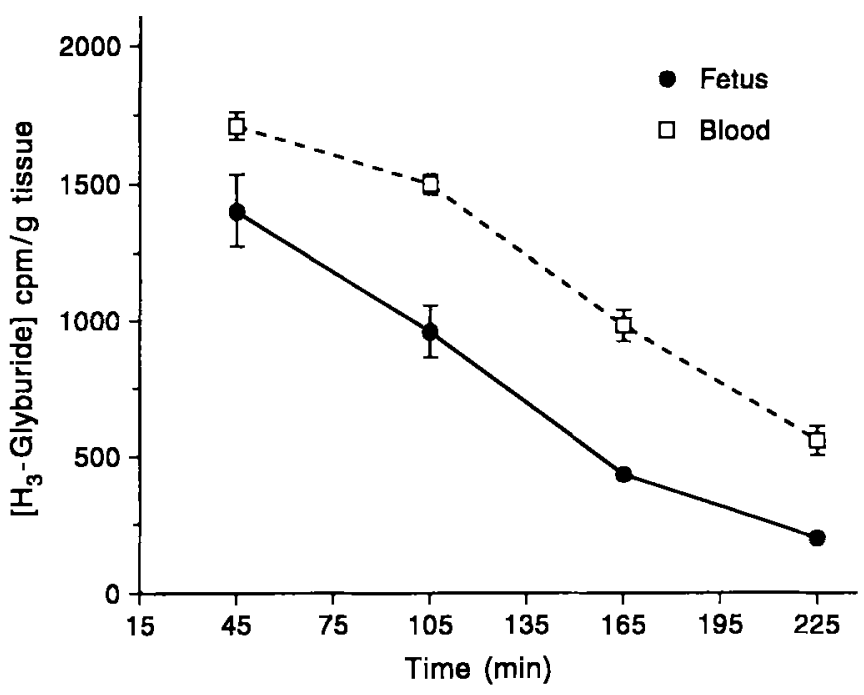

Fig.1. Time-related placental transport of glyburide. Eight pregnant rats were injected intraperitonealy with tritiated glyburide (single dose of $20 \mu \mathrm{Ci} / a n i m a l$ ). At each time point between 20 and 225 min after injection two animals were anaesthetized, bled by heart puncture and their fetuses isolated. Radioactivity of tissue homogenates and material sera were determined as described in "Materials and methods"

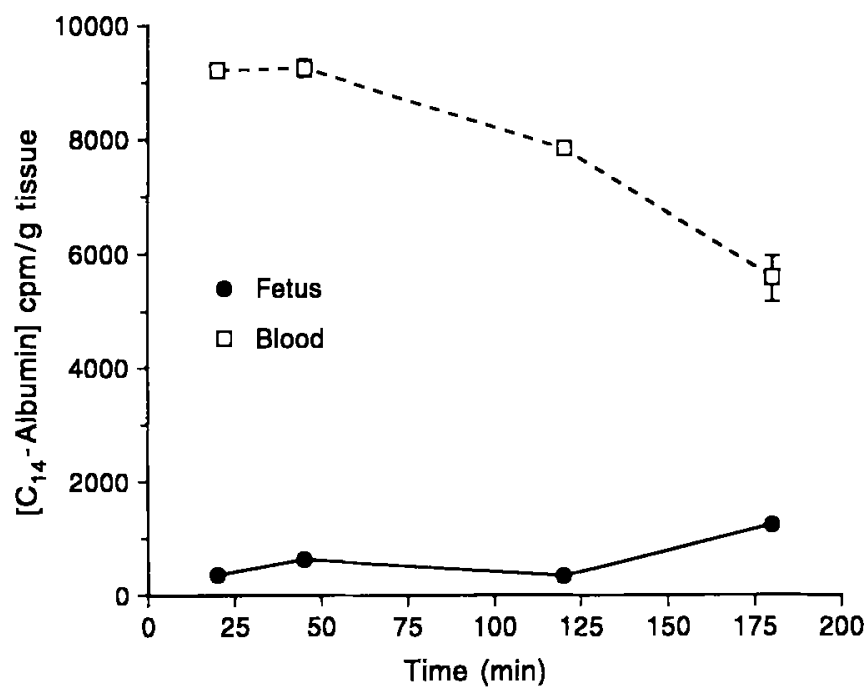

Fig. 2. Time-related placental transport of albumin. Eight pregnant rats were injected intravenously with $\mathrm{C}^{14}$-methylated albumin (single dose of $0.1 \mu \mathrm{Ci} /$ animal). At each time point between 20 and 225 min after injection two animals were anaesthetized, bled by heart puncture and their fetuses isolated. Radioactivity of tissue homogenates and material sera were determined as described in "Materials and methods"

\section{Simultaneous injection of radiolabelled glyburide and albumin}

The ratios between radioactivity in fetal tissue to those measured in maternal blood, following injection into the same animal of tritiated glyburide and $\mathrm{C}^{14}$-labelled albumin, were $0.058 \pm 0.0098$ for albumin and $0.596 \pm 0.124$ for glyburide. These results confirm the previous conclusion that glyburide is readily transported from mother to fetus and ex- cludes the possibility that the results were affected by the solvents or animal variability.

\section{Discussion}

At present OHAs are contraindicated for use during pregnancy because of potential teratogenicity and prolonged neonatal hypoglycaemia. However, published data on this issue are somewhat contradictory.

There have been a few case reports of congenital malformations associated with OHAs taken during pregnancy $[6,7]$. A number of studies have also described profound neonatal hypoglycaemia in children whose mothers took sulphonylurea during pregnancy $[8,9]$. On the other hand, reviewing the data from a number of reports describing a total of 130 diabetic mothers treated with sulphonylurea during pregnancy, no cases of severe neonatal hypoglycaemia have been found [10-12]. Furthermore, most of the adverse affects described occurred when pregnant women were treated by first-generation sulphonylureas.

It has recently been claimed by Elliot et al. [13] that glyburide, a widely-used second-generation sulphonylurea passes the placental barrier in insignificant amounts as measured in isolated single human cotelydon models. This finding ascribed to glyburides large size and configuration, and led to the suggestion that it could be safely administered during pregnancy. Our results negate this conclusion as they show glyburide to be distributed, in vivo in a fashion similar to diazepam and dissimilar to albumin, thus readily crossing the placenta.

These results support the prevailing view, recently summarized by Piacquadio et al. [14] that exposure to OHA during pregnancy may have an adverse effect on the fetus. Their report suggests that administration of OHA during embryogenesis may be teratogenic to the fetus and may cause prolonged hypoglycaemia in the neonate due to fetal hyperinsulinism and the extended action of these drugs. Our findings lead us to conclude that glyburide should be cautiously examined as a hypoglycaemic agent in the treatment of gestational diabetes due to its potentially harmful effects on the fetus and neonate.

\section{References}

1. Gerich JE (1989) Oral hypoglycemic agents. N Engl J Med 321: 1231-1245

2. London M, Gobbe S, Sach L (1990) Management of diabetes mellitus and pregnancy: a survey of obstetricians and maternal-fetal specialists. Obstet Gynecol 75: 635-640

3. Vaughn NSA (1987) Prescribing in pregnancy. Treatment of diabetes in pregnancy. BMJ 294: 558-560

4. Coustan DR (1988) Management of gestational diabetes. In: Reece EA, Coustan DR (eds) Diabetes mellitus in 
pregnancy. Principles and practice. Churchill Livingstone New York, p443

5. Adams PAJ, Schwartz R (1968) Diagnosis and treatment: should oral hypoglycemic agents be used in pediatric and pregnant patients? Pediatrics 42: 819-823

6. Larsson Y, Sterky G (1960) Possible teratogenic effect of tolbutamide in a pregnant prediabetic. Lancet, 1424-1425

7. Schiff D, Aranda JV, Stern L (1970) Neonatal thrombocytopenia and congenital malformations associated with administration of tolbutamide to the mother. J Pediatr 77: 457-458

8. Zucker P, Simon G (1968) Prolonged symptomatic neonatal hypoglycaemia associated with maternal chlorpropamide therapy. Pediatrics 42: 824825

9. Kemball ML, McIver C, Milner RDG, Nourse CYH, Schiff D, Tierman JR (1970) Neonatal hypoglycaemia in infants of diabetic mothers given sulfonylurea drugs in pregnancy. Arch Dis Child 45: 696-701
10. Coetzee EJ, Jackson WPU (1980) Pregnancy in established non-insulin-dependent diabetics. S Afr Med J 58: 795-802

11. Sutherland HW, Stowers JM, Cormack JD, Bewsher PD (1973) Evaluation of chlorpropamide in chemical diabetes diagnosed during pregnancy. BMJ 3: 9-13

12. Stowers JM, Sutherland HW (1975) The use of sulfonylureas, biguanides and insulin in pregnancy. In: Sutherland HW, Stowers JM, Persson DWM (eds) Carbohydrate metabolism in pregnancy and the newborn. Churchill Livingstone, Edinburgh, pp 205-220

13. Elliot BD, Langer O, Schenkers S, Johnson RF (1991) Insignificant transfer of glyburide occurs across the human placenta. Am J Obstet Gynecol 165: 807-812

14. Piacquadio K, Hollingsworth DR, Murphey H (1991) Effects of in-utero exposure to oral hypoglycaemic drugs. Lancet 338: 866-869 